\title{
Special Polynomials Associated with the Noumi-Yamada System of Type $A_{5}^{(1)}$
}

By

\author{
Tetsu Masuda
}

(Kobe University, Japan)

\begin{abstract}
A determinant formula for algebraic solutions to the Noumi-Yamada system of type $A_{5}^{(1)}$ is presented. This expression is regarded as a special case of the universal characters. The entries of the determinant are given by the Laguerre polynomials. Degeneration to the rational solutions to the Painlevé IV equation is discussed.

Keywords and Phrases. Noumi-Yamada system, Special polynomials, Universal characters.

2000 Mathematics Subject Classification Numbers. 33E17, 33C45, 34M55.
\end{abstract}

\section{Introduction}

Noumi and Yamada have generalized the Painlevé equations from the viewpoint of symmetry and presented higher order analogues of the Painlevé equations [7]. These systems admit the affine Weyl group symmetry of type $A_{n-1}^{(1)}$. When $n=3$ and $n=4$, the systems are nothing but the Painlevé IV equation $\left(\mathrm{P}_{\mathrm{IV}}\right)$ and the Painlevé $\mathrm{V}$ equation $\left(\mathrm{P}_{\mathrm{V}}\right)$, respectively. It is also known that they admit the special solutions expressible by the $n$-core Schur functions, which originates that the system can be derived from the $n$-reduced KP hierarchy $[8,6]$.

However, it is easy to see that the special polynomials which characterize the rational solutions to $\mathrm{P}_{\mathrm{V}}$ cannot be understood in such a picture. The author has shown that they are expressed in terms of the universal characters [2], a kind of generalization of the Schur functions [5]. In the determinant formula of Jacobi-Trudi type, the entries are given by the Laguerre polynomials.

This is not an isolated result. In fact, it has been revealed that the universal characters appear associated with a class of algebraic solutions to $\mathrm{P}_{\mathrm{VI}}$ and the Garnier systems $[4,11]$. It is also known that the rational solutions to $q-\mathrm{P}_{\mathrm{V}}$ are expressed in terms of a $q$-analogue of the universal characters [3].

Watching the construction of the rational solutions to $\mathrm{P}_{\mathrm{V}}$, one expects that the similar special polynomials appear for the cases of the Noumi-Yamada system of type $A_{2 n-1}^{(1)}(n \geq 3)$. In this article, we consider the case of $n=3$ or the Noumi-Yamada system of type $A_{5}^{(1)}$ and show that the associated special 
polynomials are expressed in terms of the universal characters specified by two 3-core partitions.

\section{The Noumi-Yamada system of type $A_{5}^{(1)}$}

The Noumi-Yamada system [7] of type $A_{5}^{(1)}$ is a differential system for unknown functions $f_{i}=f_{i}(t)(i=0,1, \ldots, 5)$ containing complex parameters $\alpha_{i}(i=0,1, \ldots, 5)$ with a constraint

$$
\alpha_{0}+\alpha_{1}+\alpha_{2}+\alpha_{3}+\alpha_{4}+\alpha_{5}=1 \text {. }
$$

The explicit formula of $f_{0}^{\prime}$ is given by

$$
\begin{aligned}
f_{0}^{\prime}= & f_{0}\left(f_{1} f_{2}+f_{1} f_{4}+f_{3} f_{4}-f_{2} f_{3}-f_{2} f_{5}-f_{4} f_{5}\right) \\
& +\left(\frac{1}{2}-\alpha_{2}-\alpha_{4}\right) f_{0}+\alpha_{0}\left(f_{2}+f_{4}\right), \quad '=t \frac{d}{d t} .
\end{aligned}
$$

Formulas of the other $f_{i}^{\prime}$ are obtained by the rotation of indices that are understood as elements of $\boldsymbol{Z} / 6 \boldsymbol{Z}$. The system is essentially fourth order since it has two trivial integrals

$$
f_{0}+f_{2}+f_{4}=f_{1}+f_{3}+f_{5}=\sqrt{t} .
$$

It is known that the system admits the extended affine Weyl group $\tilde{W}\left(A_{5}^{(1)}\right)=\left\langle s_{0}, \ldots, s_{5}, \pi\right\rangle$ as the symmetry of Bäcklund transformations. The actions are given by

$$
\begin{array}{ll}
s_{i}\left(\alpha_{i}\right)=-\alpha_{i}, & s_{i}\left(\alpha_{j}\right)=\alpha_{j}+\alpha_{i}(j=i \pm 1), \quad s_{i}\left(\alpha_{j}\right)=\alpha_{j} \quad(j \neq i, i \pm 1), \\
s_{i}\left(f_{i}\right)=f_{i}, & s_{i}\left(f_{j}\right)=f_{j} \pm \frac{\alpha_{i}}{f_{i}}(j=i \pm 1), \quad s_{i}\left(f_{j}\right)=f_{j} \quad(j \neq i, i \pm 1),
\end{array}
$$

and the fundamental relations

$$
\begin{gathered}
s_{i}^{2}=1, \quad s_{i} s_{j}=s_{j} s_{i} \quad(j \neq i, i \pm 1), \quad s_{i} s_{j} s_{i}=s_{j} s_{i} s_{j} \quad(j=i \pm 1), \\
\pi^{6}=1, \quad \pi s_{i}=s_{i+1} \pi,
\end{gathered}
$$

hold. Moreover, the actions can be lifted to the $\tau$-functions $\tau_{i}$ by the formulas

$$
s_{i}\left(\tau_{j}\right)=\tau_{j} \quad(i \neq j), \quad s_{i}\left(\tau_{i}\right)=f_{i} \frac{\tau_{i-1} \tau_{i+1}}{\tau_{i}}, \quad \pi\left(\tau_{i}\right)=\tau_{i+1} .
$$

From (2.4) and (2.6), one can derive the bilinear relations of Hirota-Miwa type

$$
\begin{aligned}
& \tau_{0} s_{0} s_{1}\left(\tau_{1}\right)=s_{0}\left(\tau_{0}\right) s_{1}\left(\tau_{1}\right)+\alpha_{0} \tau_{2} \tau_{5}, \\
& \tau_{1} s_{1} s_{0}\left(\tau_{0}\right)=s_{0}\left(\tau_{0}\right) s_{1}\left(\tau_{1}\right)-\alpha_{1} \tau_{2} \tau_{5},
\end{aligned}
$$

and their rotations of indices. 
Let us define the translation operators $T_{i}(i=0,1, \ldots, 5)$ by $T_{1}=$ $\pi s_{5} s_{4} s_{3} s_{2} s_{1}$ and $\pi T_{i}=T_{i+1} \pi$, which commute with each other and satisfy $T_{1} T_{2} T_{3} T_{4} T_{5} T_{0}=1$. The actions on the parameters $\alpha_{i}$ are given by

$$
T_{1}\left(\alpha_{0}, \alpha_{1}, \alpha_{2}, \alpha_{3}, \alpha_{4}, \alpha_{5}\right)=\left(\alpha_{0}+1, \alpha_{1}-1, \alpha_{2}, \alpha_{3}, \alpha_{4}, \alpha_{5}\right),
$$

and so on. Then a multi-index $v=\left(v_{1}, \ldots, v_{5}, v_{0}\right) \in \boldsymbol{Z}^{6}$ uniquely corresponds to an arbitrary $w \in \tilde{W}\left(A_{5}^{(1)}\right)$ by $w=T_{1}^{v_{1}} \ldots T_{5}^{v_{5}} T_{0}^{v_{0}}$. Note that all the multi-indices $v+k=\left(v_{1}+k, \ldots, v_{5}+k, v_{0}+k\right)(k \in \boldsymbol{Z})$ correspond to the same $w \in \tilde{W}\left(A_{5}^{(1)}\right)$ due to $T_{1} T_{2} T_{3} T_{4} T_{5} T_{0}=1$.

Let us introduce the $\tau$-functions on the lattice as

$$
\tau_{v}=T^{v}\left(\tau_{0}\right), \quad T^{v}=T_{1}^{v_{1}} \ldots T_{5}^{v_{5}} T_{0}^{v_{0}} .
$$

Then $\tau_{v}$ are expressed in the form

$$
\tau_{v}=\phi_{v} \tau_{0}\left(\frac{\tau_{1}}{\tau_{0}}\right)^{v_{1}} \ldots\left(\frac{\tau_{5}}{\tau_{4}}\right)^{v_{5}}\left(\frac{\tau_{0}}{\tau_{5}}\right)^{v_{0}}
$$

where $\phi_{v}$ are polynomials in $\alpha_{i}, f_{i}$ with coefficients in $Z$ and expressed by the determinant formula of Jacobi-Trudi type.

\section{Construction of special polynomials}

The similar formulation to the previous section for the Noumi-Yamada system of type $A_{n-1}^{(1)}$ is given in $[7,8,6]$. Starting with the fixed point with respect to the transformation $\pi$, one obtain a solution

$$
\alpha_{i}=\frac{1}{n}, \quad f_{i}=\frac{x}{n} \quad(i=0,1, \ldots, n-1) .
$$

The polynomials $\phi_{v}$ with the specialization of (3.1) are known to be expressed in terms of the $n$-core Schur functions. In the case of $n=3$ or $\mathrm{P}_{\mathrm{IV}}$, these polynomials coincide with the Okamoto polynomials up to multiplication by non-zero constants $[9,10]$.

As we mentioned above, the special polynomials which characterize the rational solutions to $\mathrm{P}_{\mathrm{V}}$ cannot be understood in such a picture and these polynomials are expressed in terms of the universal characters. Note that such special polynomials are constructed by starting with the fixed points with respect to the transformation $\pi^{2}$ (not $\pi$ ). It is meaningful to consider the fixed points of $\pi^{2}$ only for the cases of $A_{2 n-1}^{(1)}$. This is the reason why we investigate the Noumi-Yamada system of type $A_{5}^{(1)}$ (or $A_{2 n-1}^{(1)}$ more generally) in this article.

It is obvious that the Noumi-Yamada system of type $A_{5}^{(1)}$ has a solution 


$$
\begin{gathered}
\alpha_{2 i}=\frac{1}{3}-s, \quad \alpha_{2 i+1}=s \quad(i=0,1,2), \\
f_{i}=\frac{\sqrt{t}}{3} \quad(i=0,1, \ldots, 5),
\end{gathered}
$$

on the fixed points with respect to the transformation $\pi^{2}$. Applying the Bäcklund transformations to the above solution, we observe that $\phi_{v}$ are expressed in the form

$$
\phi_{v}=\left(\frac{\sqrt{t}}{3}\right)^{\tilde{v}(\tilde{v}-1) / 2} U_{v_{-}, v_{+}},
$$

$$
\tilde{v}=v_{1}-v_{2}+v_{3}-v_{4}+v_{5}-v_{0}, \quad v_{-}=\left(v_{1}, v_{3}, v_{5}\right), \quad v_{+}=\left(v_{2}, v_{4}, v_{0}\right),
$$

where $U_{v_{-}, v_{+}}=U_{v_{-}, v_{+}}(t, s)$ are some polynomials in $t$ and $s$. The solutions to the system are written as

$$
\begin{aligned}
& f_{0}=\frac{\sqrt{t}}{3} \frac{U_{(0,0,0)_{-}(0,0,0)_{+}} U_{(1,0,0)_{-}(0,0,-1)_{+}}}{U_{(1,1,1)_{-}(1,1,0)_{+}} U_{(1,0,0)_{-}(0,0,0)_{+}}}, \quad f_{1}=\frac{\sqrt{t}}{3} \frac{U_{(1,0,0)_{-}(0,0,0)_{+}} U_{(0,0,0)_{-}(1,0,0)_{+}}}{U_{(0,0,0)_{-}(0,0,0)_{+}} U_{(1,0,0)_{-}(1,0,0)_{+}}} \\
& f_{2}=\frac{\sqrt{t}}{3} \frac{U_{(1,0,0)_{-}(1,0,0)_{+}} U_{(1,1,0)_{-}(0,0,0)_{+}}}{U_{(1,0,0)_{-}(0,0,0)_{+}} U_{(1,1,0)_{-}(1,0,0)_{+}}}, \quad f_{3}=\frac{\sqrt{t}}{3} \frac{U_{(1,1,0)_{-}(1,0,0)_{+}} U_{(1,0,0)_{-}(1,1,0)_{+}}}{U_{(1,0,0)_{-}(1,0,0)_{+}} U_{(1,1,0)_{-}(1,1,0)_{+}}} \\
& f_{4}=\frac{\sqrt{t}}{3} \frac{U_{(1,1,0)_{-}(1,1,0)_{+}} U_{(0,0,0)_{-}(0,-1,-1)_{+}}}{U_{(1,1,0)_{-}(1,0,0)_{+}} U_{(1,1,1)_{-}(1,1,0)_{+}}}, \quad f_{5}=\frac{\sqrt{t}}{3} \frac{U_{(1,1,1)_{-}(1,1,0)_{+}} U_{(0,0,-1)_{-}(0,0,0)_{+}}}{U_{(1,1,0)_{-}(1,1,0)_{+}} U_{(0,0,0)_{-}(0,0,0)_{+}}},
\end{aligned}
$$

and

$$
\begin{array}{ll}
\alpha_{0}=\frac{1}{3}-s-v_{0}+v_{1}, & \alpha_{1}=s-v_{1}+v_{2}, \\
\alpha_{2}=\frac{1}{3}-s-v_{2}+v_{3}, & \alpha_{3}=s-v_{3}+v_{4}, \\
\alpha_{4}=\frac{1}{3}-s-v_{4}+v_{5}, & \alpha_{5}=s-v_{5}+v_{0},
\end{array}
$$

where we denote $\left(v_{1}+n_{1}, v_{3}+n_{3}, v_{5}+n_{5}\right)$ and $\left(v_{2}+n_{2}, v_{4}+n_{4}, v_{0}+n_{0}\right)$ as $\left(n_{1}, n_{3}, n_{5}\right)_{-}$and $\left(n_{2}, n_{4}, n_{0}\right)_{+}$, respectively.

We introduce some notations in order to present an explicit expression of the polynomials $U_{v_{-}, v_{+}}$. Let $M_{v_{ \pm}}$be the Maya diagrams determined by

$$
\begin{aligned}
& M_{v_{-}}=\left(3 \boldsymbol{Z}_{<v_{1}}+1\right) \cup\left(3 \boldsymbol{Z}_{<v_{3}}+2\right) \cup\left(3 \boldsymbol{Z}_{<v_{5}}+3\right), \\
& M_{v_{+}}=\left(3 \boldsymbol{Z}_{<v_{2}}+1\right) \cup\left(3 \boldsymbol{Z}_{<v_{4}}+2\right) \cup\left(3 \boldsymbol{Z}_{<v_{0}}+3\right) .
\end{aligned}
$$


To each Maya diagram $M=\left\{\ldots, m_{3}, m_{2}, m_{1}\right\} \quad\left(\cdots<m_{3}<m_{2}<m_{1}\right)$, one can associate a unique partition $\lambda=\left(\lambda_{1}, \lambda_{2}, \ldots,\right)$ such that $m_{i}-m_{i+1}=\lambda_{i}-\lambda_{i+1}+1$ for $i=1,2, \ldots$ Note that all the Maya diagrams $M+k=\left\{\ldots, m_{2}+k\right.$, $\left.m_{1}+k\right\}(k \in \boldsymbol{Z})$ obtained from $M=\left\{\ldots, m_{3}, m_{2}, m_{1}\right\}$ by shifting define the same partition by this correspondence. We assign the partitions $\lambda_{-}^{\prime}$ and $\lambda_{+}$to the Maya diagrams $M_{v_{-}}$and $M_{v_{+}}$, respectively. Note that $M_{v_{-}}$corresponds to the conjugate $\lambda_{-}^{\prime}$ and that the partitions of the form $\lambda_{ \pm}$are called 3-core. Let $H_{\lambda_{ \pm}}$be the products of the hook-length of the Young diagrams corresponding to the partitions $\lambda_{ \pm}$, which are expressed as

$$
H_{\lambda_{ \pm}}=\prod_{i \in M_{v_{ \pm}}, j \in M_{v_{ \pm}}^{c} ;>>j}(i-j) .
$$

For a pair of partitions $\lambda=\left(\lambda_{1}, \lambda_{2}, \ldots, \lambda_{l}\right)$ and $\mu=\left(\mu_{1}, \mu_{2}, \ldots, \mu_{m}\right)$, the universal character $S_{\lambda, \mu}[x, y]$ is a polynomial in $x=\left(x_{1}, x_{2}, \ldots\right)$ and $y=\left(y_{1}, y_{2}, \ldots\right)$ defined as follows [2]:

$$
S_{\lambda, \mu}[x, y]=\operatorname{det}\left[\begin{array}{ll}
q_{\mu_{m-i+1}+i-j}(y), & 1 \leq i \leq m \\
p_{\lambda_{i-m}-i+j}(x), & m+1 \leq i \leq l+m
\end{array}\right]_{1 \leq i, j \leq l+m},
$$

where $p_{k}(x)$ and $q_{k}(y)$ are the elementary Schur polynomials

$$
\sum_{k \in \boldsymbol{Z}} p_{k}(x) \eta^{k}=\exp \left(\sum_{j=1}^{\infty} x_{j} \eta^{j}\right), \quad \sum_{k \in \boldsymbol{Z}} q_{k}(y) \eta^{k}=\exp \left(\sum_{j=1}^{\infty} y_{j} \eta^{j}\right) .
$$

Then we have the following Theorem.

Theorem 3.1. The polynomials $U_{v_{-}, v_{+}}$are expressed as

$$
U_{v_{-}, v_{+}}=3^{-\left|\lambda_{-}\right|}(-3)^{-\left|\lambda_{+}\right|} H_{\lambda_{-}} H_{\lambda_{+}} S_{\lambda_{-}, \lambda_{+}},
$$

where $S_{\lambda_{-}, \lambda_{+}}=S_{\lambda_{-}, \lambda_{+}}\left[x^{-}, x^{+}\right]$are the universal characters specified by the partitions $\lambda_{-}$and $\lambda_{+}$. The variables $x^{-}=\left(x_{1}^{-}, x_{2}^{-}, \ldots\right)$ and $x^{+}=\left(x_{1}^{+}, x_{2}^{+}, \ldots\right)$ are specialized as

$$
x_{j}^{-}=\frac{t}{3}+\frac{3 s-\tilde{v}}{j}, \quad x_{j}^{+}=-\frac{t}{3}+\frac{3 s-\tilde{v}}{j} .
$$

Example. Let us take the case of $v_{-}=(1,2,0), \quad v_{+}=(3,2,0)$. The corresponding Maya diagrams are

$$
M_{v_{-}}=\{\ldots, 0,1,2,5\}, \quad M_{v_{+}}=\{\ldots, 1,2,4,5,7\} .
$$

Hence we have

$$
\lambda_{-}=(1,1)=\begin{array}{|l|}
\hline 2 \\
\hline 1
\end{array}, \quad \lambda_{+}=(2,1,1)=\begin{array}{|l|l|}
\hline 4 & 1 \\
\hline 2 & \\
\cline { 1 - 1 } &
\end{array},
$$


and

$$
H_{\lambda_{-}}=2 \cdot 1=2, \quad H_{\lambda_{+}}=4 \cdot 2 \cdot 1^{2}=8 .
$$

Thus the polynomial $U_{v_{-}, v_{+}}$is expressed as

$$
U_{v_{-}, v_{+}}=3^{-2} \times(-3)^{-4} \times 2 \times 8 \times S_{\lambda_{-}, \lambda_{+}} .
$$

The determinant formula of Jacobi-Trudi type for $S_{\lambda_{-}, \lambda_{+}}$is given by

$$
S_{\lambda_{-}, \lambda_{+}}=\left|\begin{array}{ccccc}
q_{1}^{(r)} & q_{0}^{(r)} & q_{-1}^{(r)} & q_{-2}^{(r)} & q_{-3}^{(r)} \\
q_{2}^{(r)} & q_{1}^{(r)} & q_{0}^{(r)} & q_{-1}^{(r)} & q_{-2}^{(r)} \\
p_{0}^{(r)} & p_{1}^{(r)} & p_{2}^{(r)} & p_{3}^{(r)} & p_{4}^{(r)} \\
p_{-2}^{(r)} & p_{-1}^{(r)} & p_{0}^{(r)} & p_{1}^{(r)} & p_{2}^{(r)} \\
p_{-3}^{(r)} & p_{-2}^{(r)} & p_{-1}^{(r)} & p_{0}^{(r)} & p_{1}^{(r)}
\end{array}\right|,
$$

where $p_{k}^{(r)}$ and $q_{k}^{(r)}$ are defined by

$$
\begin{gathered}
\sum_{k=0}^{\infty} p_{k}^{(r)} \eta^{k}=(1-\eta)^{-r} \exp \left(-\frac{x \eta}{1-\eta}\right), \quad p_{k}^{(r)}=0 \quad \text { for } k<0, \\
q_{k}^{(r)}(x)=p_{k}^{(r)}(-x),
\end{gathered}
$$

with $x=t / 3$ and $r=3 s-\tilde{v}=3 s+2$. We remark that $p_{k}^{(r)}$ and $q_{k}^{(r)}$ are nothing but the Laguerre polynomials.

Outline of the proof of Theorem 3.1 is given as follows. Define $R_{\lambda_{-}, \lambda_{+}}^{(r)}$ by

$$
R_{\lambda_{-}, \lambda_{+}}^{(r)}(x)=S_{\lambda_{-}, \lambda_{+}}(t, s), \quad x=\frac{t}{3}, r=3 s-\tilde{v} .
$$

Then the bilinear relations $(2.7)$ are reduced to

$$
\begin{aligned}
\sigma R_{\lambda_{-}(1,0,0) \lambda_{+}(1,0,0)}^{(r)} R_{\lambda_{-}(1,1,1) \lambda_{+}(1,1,0)}^{(r-1)} & \\
= & -R_{\lambda_{-}(0,0,0) \lambda_{+}(0,0,0)}^{(r)} R_{\lambda_{-}(1,0,0) \lambda_{+}(1,0,-1)}^{(r-1)} \\
& +R_{\lambda_{-}(1,0,0) \lambda_{+}(0,0,0)}^{(r-1)} R_{\lambda_{-}(0,0,0) \lambda_{+}(1,0,-1)}^{(r)}, \\
-\mid 3 v_{2}- & 3 v_{0}+1 \mid R_{\lambda_{-}(0,0,0) \lambda_{+}(0,0,0)}^{(r)} R_{\lambda_{-}(1,0,0) \lambda_{+}(1,0,-1)}^{(r-1)} \\
= & x R_{\lambda_{-}(1,0,0) \lambda_{+}(0,0,-1)}^{(r-2)} R_{\lambda_{-}(0,0,0) \lambda_{+}(1,0,0)}^{(r+1)} \\
& +\left(1-r-\tilde{v}-3 v_{0}+3 v_{1}\right) R_{\lambda_{-}(1,0,0) \lambda_{+}(1,0,0)}^{(r)} R_{\lambda_{-}(1,1,1) \lambda_{+}(1,1,0)}^{(r-1)},
\end{aligned}
$$


where

$$
\sigma=\left\{\begin{array}{ll}
+1 & v_{2} \geq v_{0} \\
-1 & v_{2}<v_{0}
\end{array},\right.
$$

and we denote the partitions corresponding to the multi-indices $v_{-}=\left(v_{1}+n_{1}\right.$, $\left.v_{3}+n_{3}, v_{5}+n_{5}\right)$ and $v_{+}=\left(v_{2}+n_{2}, v_{4}+n_{4}, v_{0}+n_{0}\right)$ as $\lambda_{-}\left(n_{1}, n_{3}, n_{5}\right)$ and $\lambda_{+}\left(n_{2}\right.$, $\left.n_{4}, n_{0}\right)$, respectively. The bilinear relations (3.19) can be proved in terms of the contiguity relations

$$
\begin{aligned}
& p_{k}^{(r)}-p_{k-1}^{(r)}=p_{k}^{(r-1)}, \\
& q_{k}^{(r)}-q_{k-1}^{(r)}=q_{k}^{(r-1)},
\end{aligned}
$$

and

$$
\begin{aligned}
& (k+1) p_{k+1}^{(r)}=r p_{k}^{(r+1)}-x p_{k}^{(r+2)}, \\
& (k+1) q_{k+1}^{(r)}=r q_{k}^{(r+1)}+x q_{k}^{(r+2)},
\end{aligned}
$$

by using the same technique as in [5]. For avoiding complication, we just illustrate with the case of $v_{-}=(1,2,0), v_{+}=(2,1,0)$ in Appendix.

By construction of $R_{\lambda_{-}, \lambda_{+}}^{(r)}$ we have the bilinear relations obtained from (3.19) by replacing $v_{i}$ and $R_{\lambda_{-}\left(n_{1}, n_{3}, n_{5}\right), \lambda_{+}\left(n_{2}, n_{4}, n_{0}\right)}^{(r)}$ with $v_{i+2}$ and $R_{\lambda_{-}\left(n_{5}+1, n_{1}, n_{3}\right), \lambda_{+}\left(n_{0}+1, n_{2}, n_{4}\right)}^{(r)}$, respectively. Due to the symmetry

$$
R_{\lambda_{-}, \lambda_{+}}^{(r)}(-x)=R_{\lambda_{+}, \lambda_{-}}^{(r)}(x)
$$

we also have the bilinear relations obtained by replacing $x$ and $R_{\lambda_{-}\left(n_{1}, n_{3}, n_{5}\right), \lambda_{+}\left(n_{2}, n_{4}, n_{0}\right)}^{(r)}$ by $-x$ and $R_{\lambda_{-}\left(n_{2}, n_{4}, n_{0}\right), \lambda_{+}\left(n_{1}, n_{3}, n_{5}\right)}^{(r)}$, respectively. These bilinear relations are equivalent to those derived from the rotations of indices in (2.7).

\section{Degeneration to the Okamoto polynomials}

In this section, we show that the special polynomials obtained in the previous section degenerate to the Okamoto polynomials which characterize the rational solutions to $\mathrm{P}_{\mathrm{IV}}$. This degeneration process is achieved by putting

$$
x=3 \varepsilon^{-2}\left(1-\frac{\varepsilon}{3} z\right), \quad r=3 \varepsilon^{-2},
$$

and taking the limit of $\varepsilon \rightarrow 0$. One can put $\lambda_{-}=\varnothing$ without losing generality in this limiting procedure. Then we consider the degeneration of the solutions in the form 


$$
\begin{aligned}
f_{0} & =\sqrt{\frac{x}{3}} \frac{R_{\lambda_{+}(0,0,0)}^{(r)} R_{\lambda_{+}(0,0,-1)}^{(r-2)}}{R_{\lambda_{+}(1,1,0)}^{(r-1)} R_{\lambda_{+}(0,0,0)}^{(r-1)}}, & f_{1} & =\sqrt{\frac{x}{3}} \frac{R_{\lambda_{+}(0,0,0)}^{(r-1)} R_{\lambda_{+}}^{(r+1), 0,0)}}{R_{\lambda_{+}(0,0,0)}^{(r)} R_{\lambda_{+}(1,0,0)}^{(r)}}, \\
f_{2} & =\sqrt{\frac{x}{3}} \frac{R_{\lambda_{+}(1,0,0)}^{(r)} R_{\lambda_{+}(0,0,0)}^{(r-2)}}{R_{\lambda_{+}(0,0,0)}^{(r-1)} R_{\lambda_{+}(1,0,0)}^{(r-1)}}, & f_{3} & =\sqrt{\frac{x}{3}} \frac{R_{\lambda_{+}(1,0,0)}^{(r-1)} R_{\lambda_{+}(1,1,0)}^{(r+1)}}{R_{\lambda_{+}(1,0,0)}^{(r)} R_{\lambda_{+}(1,1,0)}^{(r)}}, \\
f_{4} & =\sqrt{\frac{x}{3}} \frac{R_{\lambda_{+}(1,1,0)}^{(r)} R_{\lambda_{+}(0,-1,-1)}^{(r-2)}}{R_{\lambda_{+}(1,0,0)}^{(r)} R_{\lambda_{+}(1,1,0)}^{(r-1)}}, & f_{5} & =\sqrt{\frac{x}{3}} \frac{R_{\lambda_{+}(1,1,0)}^{(r-1)} R_{\lambda_{+}(0,0,0)}^{(r+1)}}{R_{\lambda_{+}(1,1,0)}^{(r)} R_{\lambda_{+}(0,0,0)}^{r)}},
\end{aligned}
$$

and

$$
\begin{aligned}
& \alpha_{0}=\frac{1}{3}-s-v_{0}, \quad \alpha_{1}=s+v_{2}, \\
& \alpha_{2}=\frac{1}{3}-s-v_{2}, \quad \alpha_{3}=s+v_{4}, \\
& \alpha_{4}=\frac{1}{3}-s-v_{4}, \quad \alpha_{5}=s+v_{0},
\end{aligned}
$$

with $r=3 s+\left(v_{2}+v_{4}+v_{0}\right)$. Let us investigate the degeneration of the polynomials $R_{\lambda_{+}}^{(r)}$. Putting

$$
\eta \rightarrow \varepsilon \eta, \quad \bar{p}_{k}^{(r)}=\varepsilon^{k} p_{k}^{(r)},
$$

in (3.17), we have

$$
\sum_{k=0}^{\infty} \bar{p}_{k}^{(r+j)} \eta^{k}=\exp \left(z \eta-\frac{3}{2} \eta^{2}\right)\left[1+\varepsilon\left(j \eta+z \eta^{2}-2 \eta^{3}\right)+O\left(\varepsilon^{2}\right)\right] .
$$

This implies

$$
\bar{p}_{k}^{(r+j)}=p_{k}+\varepsilon j p_{k-1}+\varepsilon\left(z p_{k-2}-2 p_{k-3}\right)+O\left(\varepsilon^{2}\right),
$$

where $p_{k}=p_{k}(z)$ are the polynomials defined by

$$
\sum_{k=0}^{\infty} p_{k} \eta^{k}=\exp \left(z \eta-\frac{3}{2} \eta^{2}\right), \quad p_{k}=0 \quad \text { for } k<0 .
$$

Then we obtain

$$
R_{\lambda_{+}}^{(r+j)}=\varepsilon^{\left|\lambda_{+}\right|}\left[R_{\lambda_{+}}+\varepsilon\left(j \frac{d R_{\lambda_{+}}}{d z}+Q_{\lambda_{+}}\right)+O\left(\varepsilon^{2}\right)\right],
$$

by using the relation 


$$
\frac{d p_{k}}{d z}=p_{k-1}
$$

where we denote the contribution of the third term of (4.6) as $Q_{\lambda_{+}}$. The polynomials $R_{\lambda_{+}}=R_{\lambda_{+}}(z)$ coincide with the Okamoto polynomials up to multiplication by non-zero constants.

Next we investigate the degeneration of the solutions and equations. It is easy to see that $f_{i}$ are expressed in the form

$$
\begin{aligned}
& f_{0}=\varepsilon^{-1}+\frac{d}{d z} \log \frac{R_{\lambda_{+}(0,0,0)}}{R_{\lambda_{+}(1,1,0)}}-\frac{z}{6}+O(\varepsilon), \quad f_{1}=\varepsilon^{-1}+\frac{d}{d z} \log \frac{R_{\lambda_{+}(1,0,0)}}{R_{\lambda_{+}(0,0,0)}}-\frac{z}{6}+O(\varepsilon), \\
& f_{2}=\varepsilon^{-1}+\frac{d}{d z} \log \frac{R_{\lambda_{+}(1,0,0)}}{R_{\lambda_{+}(0,0,0)}}-\frac{z}{6}+O(\varepsilon), \quad f_{3}=\varepsilon^{-1}+\frac{d}{d z} \log \frac{R_{\lambda_{+}(1,1,0)}}{R_{\lambda_{+}(1,0,0)}}-\frac{z}{6}+O(\varepsilon), \\
& f_{4}=\varepsilon^{-1}+\frac{d}{d z} \log \frac{R_{\lambda_{+}(1,1,0)}}{R_{\lambda_{+}(1,0,0)}}-\frac{z}{6}+O(\varepsilon), \quad f_{5}=\varepsilon^{-1}+\frac{d}{d z} \log \frac{R_{\lambda_{+}(0,0,0)}}{R_{\lambda_{+}(1,1,0)}}-\frac{z}{6}+O(\varepsilon) .
\end{aligned}
$$

Put

$$
\begin{array}{ll}
f_{0}=\varepsilon^{-1}+g_{1}-\frac{z}{2}, & f_{1}=\varepsilon^{-1}+g_{2}-\frac{z}{2}, \\
f_{2}=\varepsilon^{-1}+g_{2}-\frac{z}{2}, & f_{3}=\varepsilon^{-1}+g_{0}-\frac{z}{2}, \\
f_{4}=\varepsilon^{-1}+g_{0}-\frac{z}{2}, & f_{5}=\varepsilon^{-1}+g_{1}-\frac{z}{2},
\end{array}
$$

and

$$
\begin{array}{ll}
\alpha_{0}=-\varepsilon^{-2}+\frac{2 \beta_{0}+\beta_{1}}{3}, & \alpha_{1}=\varepsilon^{-2}+\frac{\beta_{0}-\beta_{1}}{3}, \\
\alpha_{2}=-\varepsilon^{-2}+\frac{2 \beta_{1}+\beta_{2}}{3}, & \alpha_{3}=\varepsilon^{-2}+\frac{\beta_{1}-\beta_{2}}{3}, \\
\alpha_{4}=-\varepsilon^{-2}+\frac{2 \beta_{2}+\beta_{0}}{3}, & \alpha_{5}=\varepsilon^{-2}+\frac{\beta_{2}-\beta_{0}}{3} .
\end{array}
$$

Then we find that the equations

$$
\begin{aligned}
& \left(f_{0} f_{1}\right)^{\prime}=\sqrt{t}\left[f_{0} f_{1}\left(f_{1}-f_{0}\right)+\alpha_{0} f_{1}+\alpha_{1} f_{0}\right], \\
& \left(f_{2} f_{3}\right)^{\prime}=\sqrt{t}\left[f_{2} f_{3}\left(f_{3}-f_{2}\right)+\alpha_{2} f_{3}+\alpha_{3} f_{2}\right], \\
& \left(f_{4} f_{5}\right)^{\prime}=\sqrt{t}\left[f_{4} f_{5}\left(f_{5}-f_{4}\right)+\alpha_{4} f_{5}+\alpha_{5} f_{4}\right],
\end{aligned}
$$

are reduced to 


$$
\begin{aligned}
& g_{0}^{\prime}=g_{0}\left(g_{1}-g_{2}\right)+\beta_{0}, \\
& g_{1}^{\prime}=g_{1}\left(g_{2}-g_{0}\right)+\beta_{1}, \quad,=\frac{d}{d z}, \\
& g_{2}^{\prime}=g_{2}\left(g_{0}-g_{1}\right)+\beta_{2},
\end{aligned}
$$

with

$$
\beta_{0}+\beta_{1}+\beta_{2}=1, \quad g_{0}+g_{1}+g_{2}=z,
$$

in the degeneration limit. This is nothing but the symmetric form of $\mathrm{P}_{\mathrm{IV}}$ or the Noumi-Yamada system of type $A_{2}^{(1)}$. The solutions are reduced to

$$
\begin{gathered}
g_{0}=\frac{d}{d z} \log \frac{R_{\lambda_{+}(1,1,0)}}{R_{\lambda_{+}(1,0,0)}}+\frac{z}{3}, \quad g_{1}=\frac{d}{d z} \log \frac{R_{\lambda_{+}(0,0,0)}}{R_{\lambda_{+}(1,1,0)}}+\frac{z}{3}, \\
g_{2}=\frac{d}{d z} \log \frac{R_{\lambda_{+}(1,0,0)}}{R_{\lambda_{+}(0,0,0)}}+\frac{z}{3},
\end{gathered}
$$

and

$$
\left(\beta_{0}, \beta_{1}, \beta_{2}\right)=\left(\frac{1}{3}-v_{0}+v_{2}, \frac{1}{3}-v_{2}+v_{4}, \frac{1}{3}-v_{4}+v_{0}\right),
$$

which are nothing but the rational solutions to $\mathrm{P}_{\mathrm{IV}}[9,1]$.

\section{A conjecture for the case of $A_{2 n-1}^{(1)}$}

It is easy to propose a conjecture with respect to the special polynomials associated with the Noumi-Yamada system of type $A_{2 n-1}^{(1)}$ from the discussion in the previous sections. We start with a particular solution

$$
\begin{gathered}
\alpha_{2 i}=\frac{1}{n}-s, \quad \alpha_{2 i+1}=s \quad(i=0,1, \ldots, n-1), \\
f_{i}=\frac{\sqrt{t}}{n} \quad(i=0,1, \ldots, 2 n-1),
\end{gathered}
$$

to the system on the fixed points with respect to the transformation $\pi^{2}$.

Conjecture 5.1. The functions $\phi_{v}$ with the above specialization are expressed in the form

$$
\begin{gathered}
\phi_{v}=\left(\frac{\sqrt{t}}{n}\right)^{\tilde{v}(\tilde{v}-1) / 2} U_{v_{-}, v_{+}}, \\
\tilde{v}=\sum_{i=1}^{2 n}(-1)^{i-1} v_{i}, \quad v_{-}=\left(v_{1}, \ldots, v_{2 n-1}\right), \quad v_{-}=\left(v_{2}, \ldots, v_{2 n}\right) .
\end{gathered}
$$


The polynomials $U_{v_{-}, v_{+}}=U_{v_{-}, v_{+}}(t, s)$ are expressed in terms of the universal characters as

$$
U_{v_{-}, v_{+}}=n^{-\left|\lambda_{-}\right|}(-n)^{-\left|\lambda_{+}\right|} H_{\lambda_{-}} H_{\lambda_{+}} S_{\lambda_{-}, \lambda_{+}},
$$

where $\lambda_{-}^{\prime}$ and $\lambda_{+}$are partitions of $n$-core corresponding to the Maya diagrams

$$
\begin{aligned}
& M_{v_{-}}=\left(n \boldsymbol{Z}_{<v_{1}}+1\right) \cup\left(n \boldsymbol{Z}_{<v_{3}}+2\right) \cup \cdots \cup\left(n \boldsymbol{Z}_{<v_{2 n-1}}+n\right), \\
& M_{v_{+}}=\left(n \boldsymbol{Z}_{<v_{2}}+1\right) \cup\left(n \boldsymbol{Z}_{<v_{4}}+2\right) \cup \cdots \cup\left(n \boldsymbol{Z}_{<v_{2 n}}+n\right),
\end{aligned}
$$

respectively. The variables $x^{-}$and $x^{+}$are specialized as

$$
x_{j}^{-}=\frac{t}{n}+\frac{n s-\tilde{v}}{j}, \quad x_{j}^{+}=-\frac{t}{n}+\frac{n s-\tilde{v}}{j},
$$

which means that the entries in the determinant formula of Jacobi-Trudi type are also the Laguerre polynomials.

\section{Remarks and Discussions}

It is natural to ask what kind of solutions to the Noumi-Yamada system of type $A_{5}^{(1)}$ one can get by starting with the fixed points with respect to the transformation $\pi^{3}$. In this setting, we find that the system is reduced to

$$
\begin{gathered}
f_{0}^{\prime}=\sqrt{t}\left[f_{0}\left(f_{1}-f_{2}\right)+\alpha_{0}\right], \\
f_{1}^{\prime}=\sqrt{t}\left[f_{1}\left(f_{2}-f_{3}\right)+\alpha_{1}\right], \\
f_{2}^{\prime}=\sqrt{t}\left[f_{2}\left(f_{3}-f_{0}\right)+\alpha_{2}\right], \\
\alpha_{0}+\alpha_{1}+\alpha_{2}=\frac{1}{2}, \quad f_{0}+f_{1}+f_{2}=\sqrt{t},
\end{gathered}
$$

which is equivalent to $\mathrm{P}_{\mathrm{IV}}$ or the Noumi-Yamada system of type $A_{2}^{(1)}$. It is obvious that putting $\alpha_{4}=\alpha_{5}=0$ and $f_{4}=f_{5}=0$ in the system of type $A_{5}^{(1)}$ we have

$$
\begin{aligned}
& f_{0}^{\prime}=f_{0} f_{2}\left(f_{1}-f_{3}\right)+\left(\frac{1}{2}-\alpha_{2}\right) f_{0}+\alpha_{0} f_{2}, \\
& f_{1}^{\prime}=f_{1} f_{3}\left(f_{2}-f_{0}\right)+\left(\frac{1}{2}-\alpha_{3}\right) f_{1}+\alpha_{1} f_{3}, \\
& f_{2}^{\prime}=f_{2} f_{0}\left(f_{3}-f_{1}\right)+\left(\frac{1}{2}-\alpha_{0}\right) f_{2}+\alpha_{2} f_{0}, \\
& f_{3}^{\prime}=f_{3} f_{1}\left(f_{0}-f_{2}\right)+\left(\frac{1}{2}-\alpha_{1}\right) f_{3}+\alpha_{3} f_{1},
\end{aligned}
$$




$$
\alpha_{0}+\alpha_{1}+\alpha_{2}+\alpha_{3}=1, \quad f_{0}+f_{2}=f_{1}+f_{3}=\sqrt{t},
$$

which is equivalent to $\mathrm{P}_{\mathrm{V}}$ or the Noumi-Yamada system of type $A_{3}^{(1)}$. These mean that the Noumi-Yamada system of type $A_{5}^{(1)}$ contains $\mathrm{P}_{\mathrm{IV}}$ and $\mathrm{P}_{\mathrm{V}}$ as special cases. Similarly, the solutions to the Noumi-Yamada system of type $A_{l m-1}^{(1)}(l=3,4, \ldots, m=1,2, \ldots)$ on the fixed points with respect to the transformation $\pi^{l}$ are subject to the system of type $A_{l-1}^{(1)}$.

As we mentioned above, the special polynomials associated with a class of algebraic solutions to $\mathrm{P}_{\mathrm{VI}}$ and the Garnier systems can be also expressed in terms of the universal characters. It is interesting to clarify why the universal characters appear associated with these systems. Recently, Tsuda has constructed an integrable hierarchy whose $\tau$-functions are given by the universal characters [12]. It is expected that the Noumi-Yamada system of type $A_{2 n-1}^{(1)}$ is derived as some reduction from this integrable hierarchy.

\section{A. Illustration of the proof}

In the case of $v_{-}=(1,2,0), v_{+}=(2,1,0)$, the first relation of $(3.19)$ is written as

$$
\begin{aligned}
\left|\begin{array}{ll}
q_{2}^{(r-1)} & q_{1}^{(r-1)} \\
p_{0}^{(r-1)} & p_{1}^{(r-1)}
\end{array}\right| \begin{array}{llllll}
q_{1}^{(r)} & q_{0}^{(r)} & q_{-1}^{(r)} & q_{-2}^{(r)} & q_{-3}^{(r)} & q_{-4}^{(r)} \\
q_{2}^{(r)} & q_{1}^{(r)} & q_{0}^{(r)} & q_{-1}^{(r)} & q_{-2}^{(r)} & q_{-3}^{(r)} \\
p_{2}^{(r)} & p_{3}^{(r)} & p_{4}^{(r)} & p_{5}^{(r)} & p_{6}^{(r)} & p_{7}^{(r)} \\
p_{-1}^{(r)} & p_{0}^{(r)} & p_{1}^{(r)} & p_{2}^{(r)} & p_{3}^{(r)} & p_{4}^{(r)} \\
p_{-3}^{(r)} & p_{-2}^{(r)} & p_{-1}^{(r)} & p_{0}^{(r)} & p_{1}^{(r)} & p_{2}^{(r)} \\
p_{-4}^{(r)} & p_{-3}^{(r)} & p_{-2}^{(r)} & p_{-1}^{(r)} & p_{0}^{(r)} & p_{1}^{(r)}
\end{array} \mid \\
=\left|\begin{array}{llllllll}
q_{1}^{(r)} & q_{0}^{(r)} & q_{-1}^{(r)} \\
q_{2}^{(r)} & q_{1}^{(r)} & q_{0}^{(r)} \\
p_{-1}^{(r)} & p_{0}^{(r)} & p_{1}^{(r)}
\end{array}\right| \begin{array}{llllll}
q_{2}^{(r-1)} & q_{1}^{(r-1)} & q_{0}^{(r-1)} & q_{-1}^{(r-1)} & q_{-2}^{(r-1)} \\
p_{3}^{(r-1)} & p_{4}^{(r-1)} & p_{5}^{(r-1)} & p_{6}^{(r-1)} & p_{7}^{(r-1)} \\
p_{0}^{(r-1)} & p_{1}^{(r-1)} & p_{2}^{(r-1)} & p_{3}^{(r-1)} & p_{4}^{(r-1)} \\
p_{-2}^{(r-1)} & p_{-1}^{(r-1)} & p_{0}^{(r-1)} & p_{1}^{(r-1)} & p_{2}^{(r-1)} \\
p_{-3}^{(r-1)} & p_{-2}^{(r-1)} & p_{-1}^{(r-1)} & p_{0}^{(r-1)} & p_{1}^{(r-1)}
\end{array} \mid \\
+\left|\begin{array}{lllllll}
(r-1) \\
q_{1}^{(r-1)} & q_{0}^{(r-1)} & q_{-1}^{(r-1)} & q_{-2}^{(r-1)} & q_{-3}^{(r-1)} \\
q_{2}^{(r-1)} & q_{1}^{(r-1)} & q_{0}^{(r-1)} & q_{-1}^{(r-1)} & q_{-2}^{(r-1)} \\
p_{2}^{(r)} & q_{1}^{(r)} & q_{0}^{(r)} & p_{4}^{(r)} \\
p_{-1}^{(r)} & p_{0}^{(r)} & p_{1}^{(r)}
\end{array}\right| \begin{array}{llllll}
(r-1) & p_{1}^{(r-1)} & p_{2}^{(r-1)} & p_{3}^{(r-1)} & p_{4}^{(r-1)} \\
p_{-2}^{(r-1)} & p_{-1}^{(r-1)} & p_{0}^{(r-1)} & p_{1}^{(r-1)} & p_{2}^{(r-1)} \\
p_{-3}^{(r-1)} & p_{-2}^{(r-1)} & p_{-1}^{(r-1)} & p_{0}^{(r-1)} & p_{1}^{(r-1)}
\end{array} \mid .
\end{aligned}
$$


Using the contiguity relation (3.21), we have

(A.2)

$$
\begin{aligned}
\left|\begin{array}{llllll}
q_{1}^{(r)} & q_{0}^{(r)} & q_{-1}^{(r)} & q_{-2}^{(r)} & q_{-3}^{(r)} & q_{-4}^{(r)} \\
q_{2}^{(r)} & q_{1}^{(r)} & q_{0}^{(r)} & q_{-1}^{(r)} & q_{-2}^{(r)} & q_{-3}^{(r)} \\
p_{2}^{(r)} & p_{3}^{(r)} & p_{4}^{(r)} & p_{5}^{(r)} & p_{6}^{(r)} & p_{7}^{(r)} \\
p_{-1}^{(r)} & p_{0}^{(r)} & p_{1}^{(r)} & p_{2}^{(r)} & p_{3}^{(r)} & p_{4}^{(r)} \\
p_{-3}^{(r)} & p_{-2}^{(r)} & p_{-1}^{(r)} & p_{0}^{(r)} & p_{1}^{(r)} & p_{2}^{(r)} \\
p_{-4}^{(r)} & p_{-3}^{(r)} & p_{-2}^{(r)} & p_{-1}^{(r)} & p_{0}^{(r)} & p_{1}^{(r)}
\end{array}\right| \\
= \\
=\left|\begin{array}{lllllll}
(r) & & \\
-q_{1}^{(r)} & q_{1}^{(r-1)} & q_{0}^{(r-1)} & q_{-1}^{(r-1)} & q_{-2}^{(r-1)} & q_{-3}^{(r-1)} \\
-q_{2}^{(r)} & q_{2}^{(r-1)} & q_{1}^{(r-1)} & q_{0}^{(r-1)} & q_{-1}^{(r-1)} & q_{-2}^{(r-1)} \\
p_{2}^{(r)} & p_{3}^{(r-1)} & p_{4}^{(r-1)} & p_{5}^{(r-1)} & p_{6}^{(r-1)} & p_{7}^{(r-1)} \\
p_{-1}^{(r)} & p_{0}^{(r-1)} & p_{1}^{(r-1)} & p_{2}^{(r-1)} & p_{3}^{(r-1)} & p_{4}^{(r-1)} \\
p_{-3}^{(r)} & p_{-2}^{(r-1)} & p_{-1}^{(r-1)} & p_{0}^{(r-1)} & p_{1}^{(r-1)} & p_{2}^{(r-1)} \\
p_{-4}^{(r)} & p_{-3}^{(r-1)} & p_{-2}^{(r-1)} & p_{-1}^{(r-1)} & p_{0}^{(r-1)} & p_{1}^{(r-1)}
\end{array}\right| .
\end{aligned}
$$

Put

$$
D=\left|\begin{array}{cccccc}
-q_{1}^{(r)} & q_{1}^{(r-1)} & q_{0}^{(r-1)} & q_{-1}^{(r-1)} & q_{-2}^{(r-1)} & q_{-3}^{(r-1)} \\
-q_{2}^{(r)} & q_{2}^{(r-1)} & q_{1}^{(r-1)} & q_{0}^{(r-1)} & q_{-1}^{(r-1)} & q_{-2}^{(r-1)} \\
p_{2}^{(r)} & p_{3}^{(r-1)} & p_{4}^{(r-1)} & p_{5}^{(r-1)} & p_{6}^{(r-1)} & p_{7}^{(r-1)} \\
p_{-1}^{(r)} & p_{0}^{(r-1)} & p_{1}^{(r-1)} & p_{2}^{(r-1)} & p_{3}^{(r-1)} & p_{4}^{(r-1)} \\
p_{-3}^{(r)} & p_{-2}^{(r-1)} & p_{-1}^{(r-1)} & p_{0}^{(r-1)} & p_{1}^{(r-1)} & p_{2}^{(r-1)} \\
p_{-4}^{(r)} & p_{-3}^{(r-1)} & p_{-2}^{(r-1)} & p_{-1}^{(r-1)} & p_{0}^{(r-1)} & p_{1}^{(r-1)}
\end{array}\right|
$$

Then Jacobi's identity

$$
D \cdot D\left[\begin{array}{ll}
1 & 3 \\
1 & 6
\end{array}\right]=D\left[\begin{array}{l}
1 \\
1
\end{array}\right] D\left[\begin{array}{l}
3 \\
6
\end{array}\right]-D\left[\begin{array}{l}
1 \\
6
\end{array}\right] D\left[\begin{array}{l}
3 \\
1
\end{array}\right]
$$

is reduced to (A.1). The second relation of (3.19) is written as

$$
\left(\begin{array}{lllll}
q_{2}^{(r-2)} & q_{1}^{(r-2)} & q_{0}^{(r-2)} & q_{-1}^{(r-2)} \\
p_{1}^{(r-2)} & p_{2}^{(r-2)} & p_{3}^{(r-2)} & p_{4}^{(r-2)} \\
p_{-1}^{(r-2)} & p_{0}^{(r-2)} & p_{1}^{(r-2)} & p_{2}^{(r-2)} \\
p_{-2}^{(r-2)} & p_{-1}^{(r-2)} & p_{0}^{(r-2)} & p_{1}^{(r-2)}
\end{array}|| \begin{array}{cccc}
q_{1}^{(r+1)} & q_{0}^{(r+1)} & q_{-1}^{(r+1)} & q_{-2}^{(r+1)} \\
q_{2}^{(r+1)} & q_{1}^{(r+1)} & q_{0}^{(r+1)} & q_{-1}^{(r+1)} \\
p_{1}^{(r+1)} & p_{2}^{(r+1)} & p_{3}^{(r+1)} & p_{4}^{(r+1)} \\
p_{-2}^{(r+1)} & p_{-1}^{(r+1)} & p_{0}^{(r+1)} & p_{1}^{(r+1)}
\end{array} \mid\right.
$$




$$
\begin{gathered}
=-7\left|\begin{array}{lll}
q_{1}^{(r)} & q_{0}^{(r)} & q_{-1}^{(r)} \\
q_{2}^{(r)} & q_{1}^{(r)} & q_{0}^{(r)} \\
p_{-1}^{(r)} & p_{0}^{(r)} & p_{1}^{(r)}
\end{array}\right|\left|\begin{array}{llllll}
q_{2}^{(r-1)} & q_{1}^{(r-1)} & q_{0}^{(r-1)} & q_{-1}^{(r-1)} & q_{-2}^{(r-1)} \\
p_{3}^{(r-1)} & p_{4}^{(r-1)} & p_{5}^{(r-1)} & p_{6}^{(r-1)} & p_{7}^{(r-1)} \\
p_{0}^{(r-1)} & p_{1}^{(r-1)} & p_{2}^{(r-1)} & p_{3}^{(r-1)} & p_{4}^{(r-1)} \\
p_{-2}^{(r-1)} & p_{-1}^{(r-1)} & p_{0}^{(r-1)} & p_{1}^{(r-1)} & p_{2}^{(r-1)} \\
p_{-3}^{(r-1)} & p_{-2}^{(r-1)} & p_{-1}^{(r-1)} & p_{0}^{(r-1)} & p_{1}^{(r-1)}
\end{array}\right| \\
+(r-4)\left|\begin{array}{lllllll}
(r-1) & & & \\
q_{2}^{(r)} & q_{1}^{(r)} & q_{0}^{(r)} \\
p_{2}^{(r)} & p_{3}^{(r)} & p_{4}^{(r)} \\
p_{-1}^{(r)} & p_{0}^{(r)} & p_{1}^{(r)}
\end{array}\right| \begin{array}{lllll}
q_{1}^{(r-1)} & q_{0}^{(r-1)} & q_{-1}^{(r-1)} & q_{-2}^{(r-1)} & q_{-3}^{(r-1)} \\
q_{2}^{(r-1)} & q_{1}^{(r-1)} & q_{0}^{(r-1)} & q_{-1}^{(r-1)} & q_{-2}^{(r-1)} \\
p_{0}^{(r-1)} & p_{1}^{(r-1)} & p_{2}^{(r-1)} & p_{3}^{(r-1)} & p_{4}^{(r-1)} \\
p_{-2}^{(r-1)} & p_{-1}^{(r-1)} & p_{0}^{(r-1)} & p_{1}^{(r-1)} & p_{2}^{(r-1)} \\
p_{-3}^{(r-1)} & p_{-2}^{(r-1)} & p_{-1}^{(r-1)} & p_{0}^{(r-1)} & p_{1}^{(r-1)}
\end{array} \mid .
\end{gathered}
$$

Using the contiguity relation (3.21), we get

(A.6) $\left|\begin{array}{llll}q_{1}^{(r+1)} & q_{0}^{(r+1)} & q_{-1}^{(r+1)} & q_{-2}^{(r+1)} \\ q_{2}^{(r+1)} & q_{1}^{(r+1)} & q_{0}^{(r+1)} & q_{-1}^{(r+1)} \\ p_{1}^{(r+1)} & p_{2}^{(r+1)} & p_{3}^{(r+1)} & p_{4}^{(r+1)} \\ p_{-2}^{(r+1)} & p_{-1}^{(r+1)} & p_{0}^{(r+1)} & p_{1}^{(r+1)}\end{array}\right|=\left|\begin{array}{cccc}q_{1}^{(r-2)} & q_{0}^{(r-1)} & q_{-1}^{(r)} & q_{-2}^{(r+1)} \\ q_{2}^{(r-2)} & q_{1}^{(r-1)} & q_{0}^{(r)} & q_{-1}^{(r+1)} \\ -p_{4}^{(r-2)} & p_{4}^{(r-1)} & -p_{4}^{(r)} & p_{4}^{(r+1)} \\ -p_{1}^{(r-2)} & p_{1}^{(r-1)} & -p_{1}^{(r)} & p_{1}^{(r+1)}\end{array}\right|$.

Similarly we have

(A.7)

$$
\begin{gathered}
\left|\begin{array}{llll}
q_{1}^{(r+1)} & q_{0}^{(r+1)} & q_{-1}^{(r+1)} & q_{-2}^{(r+1)} \\
q_{2}^{(r+1)} & q_{1}^{(r+1)} & q_{0}^{(r+1)} & q_{-1}^{(r+1)} \\
p_{1}^{(r+1)} & p_{2}^{(r+1)} & p_{3}^{(r+1)} & p_{4}^{(r+1)} \\
p_{-2}^{(r+1)} & p_{-1}^{(r+1)} & p_{0}^{(r+1)} & p_{1}^{(r+1)}
\end{array}\right| \\
=\left|\begin{array}{cccccc}
q_{1}^{(r-4)} & q_{0}^{(r-3)} & q_{-1}^{(r-2)} & q_{-2}^{(r-1)} & q_{-3}^{(r)} & q_{-4}^{(r+1)} \\
q_{2}^{(r-4)} & q_{1}^{(r-3)} & q_{0}^{(r-2)} & q_{-1}^{(r-1)} & q_{-2}^{(r)} & q_{-3}^{(r+1)} \\
-p_{6}^{(r-4)} & p_{6}^{(r-3)} & -p_{6}^{(r-2)} & p_{6}^{(r-1)} & -p_{6}^{(r)} & p_{6}^{(r+1)} \\
-p_{3}^{(r-4)} & p_{3}^{(r-3)} & -p_{3}^{(r-2)} & p_{3}^{(r-1)} & -p_{3}^{(r)} & p_{3}^{(r+1)} \\
-p_{1}^{(r-4)} & p_{1}^{(r-3)} & -p_{1}^{(r-2)} & p_{1}^{(r-1)} & -p_{1}^{(r)} & p_{1}^{(r+1)} \\
-p_{0}^{(r-4)} & p_{0}^{(r-3)} & -p_{0}^{(r-2)} & p_{0}^{(r-1)} & -p_{0}^{(r)} & p_{0}^{(r+1)}
\end{array}\right|
\end{gathered}
$$

Using the contiguity relation (3.22), we obtain 
(A.8)

$$
(\mathrm{A} .7)=x^{-5}(r-4)(r-3) \times 7 \cdot 4 \cdot 2 \cdot 1
$$

$$
\times\left|\begin{array}{cccccc}
\frac{q_{1}^{(r-4)}}{r-4} & q_{1}^{(r-5)} & q_{0}^{(r-4)} & q_{-1}^{(r-3)} & q_{-2}^{(r-2)} & q_{-3}^{(r-1)} \\
\frac{q_{2}^{(r-4)}}{r-3} & q_{2}^{(r-5)} & q_{1}^{(r-4)} & q_{0}^{(r-3)} & q_{-1}^{(r-2)} & q_{-2}^{(r-1)} \\
-\frac{p_{6}^{(r-4)}}{7} & -p_{7}^{(r-5)} & p_{7}^{(r-4)} & -p_{7}^{(r-3)} & p_{7}^{(r-2)} & -p_{7}^{(r-1)} \\
-\frac{p_{3}^{(r-4)}}{4} & -p_{4}^{(r-5)} & p_{4}^{(r-4)} & -p_{4}^{(r-3)} & p_{4}^{(r-2)} & -p_{4}^{(r-1)} \\
-\frac{p_{1}^{(r-4)}}{2} & -p_{2}^{(r-5)} & p_{2}^{(r-4)} & -p_{2}^{(r-3)} & p_{2}^{(r-2)} & -p_{2}^{(r-1)} \\
-p_{0}^{(r-4)} & -p_{1}^{(r-5)} & p_{1}^{(r-4)} & -p_{1}^{(r-3)} & p_{1}^{(r-2)} & -p_{1}^{(r-1)}
\end{array}\right| .
$$

Put

$$
D=\left|\begin{array}{cccccc}
\frac{q_{1}^{(r-4)}}{r-4} & q_{1}^{(r-5)} & q_{0}^{(r-4)} & q_{-1}^{(r-3)} & q_{-2}^{(r-2)} & q_{-3}^{(r-1)} \\
\frac{q_{2}^{(r-4)}}{r-3} & q_{2}^{(r-5)} & q_{1}^{(r-4)} & q_{0}^{(r-3)} & q_{-1}^{(r-2)} & q_{-2}^{(r-1)} \\
-\frac{p_{6}^{(r-4)}}{7} & -p_{7}^{(r-5)} & p_{7}^{(r-4)} & -p_{7}^{(r-3)} & p_{7}^{(r-2)} & -p_{7}^{(r-1)} \\
-\frac{p_{3}^{(r-4)}}{4} & -p_{4}^{(r-5)} & p_{4}^{(r-4)} & -p_{4}^{(r-3)} & p_{4}^{(r-2)} & -p_{4}^{(r-1)} \\
-\frac{p_{1}^{(r-4)}}{2} & -p_{2}^{(r-5)} & p_{2}^{(r-4)} & -p_{2}^{(r-3)} & p_{2}^{(r-2)} & -p_{2}^{(r-1)} \\
-p_{0}^{(r-4)} & -p_{1}^{(r-5)} & p_{1}^{(r-4)} & -p_{1}^{(r-3)} & p_{1}^{(r-2)} & -p_{1}^{(r-1)}
\end{array}\right| .
$$

Then Jacobi's identity (A.4) is reduced to (A.5).

\section{References}

[1] Kajiwara, K. and Ohta, Y., Determinant structure of the rational solutions for the Painlevé IV equation, J. Phys. A: Math. Gen., 31 (1998), 2431-2446.

[2] Koike, K., On the decomposition of tensor products of the representations of the classical groups: by means of the universal characters, Adv. Math., 74 (1989), 57-86.

[3] Masuda, T., On the rational solutions of $q$-Painlevé V equation, Nagoya Math. J., 169 (2003), 119-143.

[4] Masuda, T., On a class of algebraic solutions to the Painlevé VI equation, its determinant formula and coalescence cascade, Funkcial. Ekvac., 46 (2003), 121-171. 
[5] Masuda, T., Ohta, Y. and Kajiwara, K., A determinant formula for a class of rational solutions of Painlevé V equation, Nagoya Math. J., 168 (2002), 1-25.

[6] Noumi, M., Painlevé equations through symmetry, Translations of Mathematical Monographs Vol. 223, American Mathematical Society, 2004.

[7] Noumi, M. and Yamada, Y., Higher order Painlevé equations of type $A_{l}^{(1)}$, Funkcial. Ekvac., 41 (1998), 483-503.

[8 ] Noumi, M. and Yamada, Y., Affine Weyl groups, discrete dynamical systems and Painlevé equations, Commun. Math. Phys., 199 (1998), 281-295.

[9] Noumi, M. and Yamada, Y., Symmetries in the fourth Painlevé equation and Okamoto polynomials, Nagoya Math. J., 153 (1999), 53-86.

[10] Okamoto, K., Studies on the Painlevé equations III, second and fourth Painlevé equations, $\mathrm{P}_{\mathrm{II}}$ and $\mathrm{P}_{\mathrm{IV}}$, Math. Ann., 275 (1986), 222-254.

[11] Tsuda, T., Toda equation and special polynomials associated with the Garnier system, preprint.

[12] Tsuda, T., Universal characters and an extension of the KP hierarchy, Comm. Math. Phys., 248 (2004), 501-526.

\author{
nuna adreso: \\ Department of Mathematics \\ Kobe University \\ Rokko, Kobe, 657-8501 \\ Japan \\ E-mail: masuda@math.kobe-u.ac.jp
}

(Ricevita la 1-an de aprilo, 2004)

(Reviziita la 3-an de septembro, 2004) 